\title{
GABAergic Inhibition in Nucleus Magnocellularis: Implications for Phase Locking in the Avian Auditory Brainstem
}

\author{
Pablo Monsivais, Lichuan Yang, and Edwin W Rubel \\ Virginia Merrill Bloedel Hearing Research Center and Department of Otolaryngology, Head and Neck Surgery, University \\ of Washington, Seattle, Washington 98195
}

In the avian auditory brainstem, nucleus magnocellularis (NM) functions to relay phase-locked signals to nucleus laminaris for binaural coincidence detection. Although many studies have revealed that NM neurons exhibit intrinsic physiological and anatomical specializations for this purpose, the role of inhibition has not been fully explored. The present study characterizes the organization of GABAergic feedback to NM. Anterograde and retrograde labeling methods showed that NM receives a prominent projection from the ipsilateral superior olivary nucleus (SON). The functional features of this projection were explored in a brain slice preparation. Stimulating fibers from the SON evoked long-lasting, depolarizing responses in NM neurons that were blockable by bicuculline, a $\mathrm{GABA}_{\mathrm{A}}$ receptor antagonist. The slow time course of these responses allowed them to undergo temporal summation during repetitive stimu-

In the avian auditory brainstem, auditory nerve fibers bif urcate to innervate neurons of two spatially segregated cochlear nuclei: nucleus angularis (NA) and nucleus magnocellularis (NM) (Boord, 1969; Ramón y Cajal, 1971; Rubel and Parks, 1975; Parks and Rubel, 1978; Jhaveri and Morest, 1982a,b; Carr and Boudreau, 1991). NM, the avian homolog of the mammalian anteroventral cochlear nucleus, projects bilaterally to nucleus laminaris (NL). By preserving temporal characteristics of acoustic inputs, NM neurons provide the information necessary for coincidence detection of interaural time differences (Young and Rubel, 1983; Sullivan and Konishi, 1984; Carr and Konishi, 1988, 1990; Warchol and Dallos, 1990; Fujita and Konishi, 1991; Overholt et al., 1992; Joseph and Hyson, 1993).

Many anatomical and physiological features make NM neurons well suited for coding temporal information. First, excitatory transmission to NM cells is very secure. Each NM neuron receives two or three large calycine terminals, each of which can generate suprathreshold currents (Parks and Rubel, 1978; Hackett et al., 1982; Zhang and Trussell, 1994a,b). Second, excitatory input is not attenuated or electrotonically filtered because NM neurons have spherical somata and few if any dendritic processes (Parks 1981; Jhaveri \& Morest, 1982a; Reyes et al., 1994; Zhang

Received Sept. 15, 1999; revised Jan. 28, 2000; accepted Feb. 1, 2000.

This work was sponsored by National Institutes of Health Grant 00395, a National Institutes of Health Neuroscience Training Grant 5T 32 GM 07108, and Fellowship DC00312. We are grateful to Drs. W. Lippe, G. Pollak, and W. Spain for critically reading and commenting on early versions of this manuscript and to M. Mazurek for many valuable discussions.

Correspondence should be addressed to Edwin W Rubel, Virginia Merrill Bloedel Hearing Research Center and Department of Otolaryngology, Head and Neck Surgery, University of Washington, Box 357923, Seattle, WA 98195. E-mail: rubel@u.washington.edu.

Copyright (C) 2000 Society for Neuroscience $\quad 0270-6474 / 00 / 202954-10 \$ 15.00 / 0$ lation. The summed GABAergic response was capable of blocking spikes generated in NM neurons by suprathreshold current injection. This inhibitory effect was attributable to a large reduction in input resistance caused by a combination of the opening of a GABAergic $\mathrm{Cl}^{-}$conductance and the recruitment of a low-voltage activated $\mathrm{K}^{+}$conductance. This large reduction of input resistance increased the amount of current necessary to drive NM neurons to threshold. The results lead us to propose that GABAergic inhibition enhances phase-locking fidelity of NM neurons, which is essential to binaural coincidence detection in nucleus laminaris.

Key words: GABA; nucleus magnocellularis; avian auditory brainstem; phase locking; superior olivary nucleus; $\mathrm{K}^{+}$ conductance

and Trussell, 1994a). Third, NM neurons express a complement of channels for rapid signaling. For instance, a robust low voltageactivated (LVA) $\mathrm{K}^{+}$conductance activates at membrane potentials just positive to rest. $\mathrm{LVA} \mathrm{K}^{+}$currents are rapid to activate and only slowly inactivate, allowing NM neurons to recover rapidly from excitation and preventing temporal summation of multiple inputs (Reyes et al., 1994; Koyano et al., 1996; Rathouz and Trussell, 1998). Furthermore, ionotropic glutamate receptors on NM neurons desensitize rapidly, keeping excitatory synaptic currents brief despite their large size (Raman and Trussell, 1992; Trussell et al., 1993; Raman et al., 1994).

NM neurons also receive inhibitory inputs. Electron microscopy reveals punctate endings containing round vesicles at symmetrical membrane specializations, characteristics of inhibitory terminals (Parks, 1981). Immunohistochemical studies have shown that these terminals are GABA-positive (Müller, 1987; Carr et al., 1989; Code et al., 1989, 1991; von Bartheld et al., 1989; Lachica et al., 1994). Furthermore, early in vitro studies on NM identified two types of evoked synaptic potentials: short latency, rapid EPSPs and longer latency and long duration depolarizing PSPs (Hackett et al., 1982). More recent in vitro studies have further confirmed the presence of GABA receptors on NM neurons; NM neurons respond to GABA directly by depolarization, and $\mathrm{Cl}^{-}$appears to be the ion mediating this response (Hyson et al., 1995; Lu et al., 1997). The depolarizing nature of GABAergic responses is presumably attributable to relatively high intracellular $\left[\mathrm{Cl}^{-}\right]$of $\mathrm{NM}$ neurons.

The origin of GABAergic input to NM neurons has only been briefly described. Besides the contribution of a small number of local GABAergic neurons, NM receives a large projection from the superior olivary nucleus (SON), which is mainly composed of 
GABAergic neurons (von Bartheld et al., 1989; Lachica et al., 1994; Westerberg and Schwarz, 1995). In addition to its NM projection, SON has reciprocal connections with NA and NL (Takahashi and Konishi, 1988; Carr et al., 1989; Carr and Boudreau, 1993; Lachica et al., 1994). Because of this connectivity and its GABAergic nature, it has been suggested that the SON functions as a "gain-control" system, especially under intense acoustic input (Lachica et al., 1994; Peña et al., 1996; Yang et al., 1999).

The present study addresses two issues: first, whether the connections between the SON and NA, NL, and NM are unilateral (ipsilateral) or bilateral; second, how the SON input affects the physiological properties of NM neurons. We demonstrated that the SON projects only to the ipsilateral NM, NA, and NL and that direct stimulation of SON input evokes a GABAergic inhibition that profoundly affected the firing properties of NM neurons. The results lead us to propose that by dampening the excitability of NM neurons, inhibition from the SON could improve their phase locking, which may be essential to binaural coincidence detection in the avian auditory brainstem.

\section{MATERIALS AND METHODS}

Experiments were conducted on White Leghorn chicken hatchlings (2- to 3-weeks-old) and late stage embryos (20- to 21-d-old). Animals were obtained from $\mathrm{H} \& \mathrm{~N}$ Farms (Redmond, WA). Hatchlings were used to study the connectivity between the SON, NM, and other auditory nuclei in the brainstem. Embryos were used for in vitro physiological investigation in brain slice preparations. Most of the techniques have been described previously (Hyson and Rubel, 1989; Overholt et al., 1992; Lachica et al., 1994; Reyes et al., 1994, 1996; Yang et al., 1999). All methods were approved by the University of Washington Animal Care Committee.

Surgical procedures and tracer injection. Thirty-five hatchling chickens were used to characterize the afferent and efferent connections of the SON. The animals were anesthetized by intraperitoneal injection of ketamine $(8 \mathrm{mg} / 100 \mathrm{gm})$ and Nembutal $(1.8 \mathrm{mg} / 100 \mathrm{gm})$. Supplemental doses were administrated at half the original dose every $60 \mathrm{~min}$. The feathers on the head were trimmed short and then removed with a depilatory. The head was then placed on a stereotaxic apparatus through a beak holder. The skin and muscles overlying the skull were reflected, and a foundation layer of cyanoacrylate and dental cement was created on the surface of the skull. The foundation was then connected to a metal bar for the fixation of the head on the stereotaxic apparatus.

A reference point was marked along the midline of the skull. Subsequently, a 2-mm-diameter hole was drilled to expose the underlying cerebellum. A glass micropipette filled with $1 \mathrm{M} \mathrm{NaCl}$ was advanced by a micromanipulator through the cerebellum and into the auditory brainstem. During electrode penetration, $20 \mathrm{msec}$ duration of pure tones of varying frequency and intensity were presented continuously. Because each auditory nucleus (namely, SON, NA, NL, and NM) had characteristic response properties (latency, discharge pattern, and relative location), they could be readily identified through multiple penetrations. After brief characterization of the nucleus, the micropipette was replaced with an injection electrode with a tip diameter of $20 \mu \mathrm{m}$, filled with $10 \%$ lysine-fixable dextran conjugated to tetramethylrhodamine ( 3 $\mathrm{kDa}, \mathrm{pH}$ 7.4; Molecular Probes, Eugene, OR). The tracer was iontophoresed into the nucleus by passing $5 \mu \mathrm{A}$ of on-off positive current for $20 \mathrm{~min}$.

After a survival time of $24-72 \mathrm{hr}$, animals were deeply anesthetized with a lethal dose of Nembutal, then perfused transcardially with PBS $(0.1 \mathrm{M}, \mathrm{pH} 7.4)$ followed by a fixative solution of $4 \%$ paraformaldehyde in PBS. After fixation, the brain was dissected out, post-fixed for $2 \mathrm{hr}$, and then transferred to a $30 \%$ sucrose solution overnight for cryoprotection. The brain was sectioned on a freezing microtome at a thickness of $40 \mu \mathrm{m}$. Sections were mounted on slides, coverslipped with Dako (Carpinteria, CA) glycergel mounting medium, and examined under light microscope with epi-illumination through a rhodamine filter cube.

Brain slice preparation and whole-cell recording. Chicken embryos were rapidly decapitated. A $4 \mathrm{~mm}$ segment of the skull containing the brainstem was removed with a razor blade and quickly submerged in ice-cold artificial CSF (ACSF). ACSF contained (in mM): $130 \mathrm{NaCl}, 26$
$\mathrm{NaH}_{2} \mathrm{CO}_{3}, 3 \mathrm{KCl}, 2 \mathrm{CaCl}_{2}, 1 \mathrm{MgCl}_{2}, 1.25 \mathrm{NaH}_{2} \mathrm{PO}_{4}$, and 10 dextrose. The brainstem segment was dissected out and transferred to a Vibratome tissue slicer (Pelco, St. Louis, MO) where it was mounted on an agar block with cyanoacrylate glue and cut in ice-cold ACSF. Several $150 \mu \mathrm{m}$ coronal slices containing the SON, NA, NL, and NM were collected and incubated in a holding chamber filled with ACSF at room temperature $\left(22-23^{\circ} \mathrm{C}\right)$. ACSF was constantly gassed with $95 \% \mathrm{O}_{2}$ and $5 \% \mathrm{CO}_{2}$ and had a $\mathrm{pH}$ of 7.4.

Slices were transferred to a $0.5 \mathrm{cc}$ volume recording chamber mounted on a Zeiss Axioskop FS with a $40 \times$ water-immersion objective and infrared, differential interference contrast optics (Zeiss, Oberkochen, Germany) and superfused with ACSF at a rate of $3 \mathrm{ml} / \mathrm{min}$. Using a multiple valve system, a slice was perfused with normal ACSF or ACSF containing $50 \mu \mathrm{M}$ bicuculline methiodide (Sigma, St. Louis, MO) or ACSF with 6,7-dinitroquinoxaline-2,3-dione (DNQX; $100 \mu \mathrm{M}$ ) and D,L2-amino-5-phosphonovalerate (AP-V; $50 \mu \mathrm{M}$ ) (Research Biochemicals, Natick, MA). All recordings were performed at room temperature $\left(22-23^{\circ} \mathrm{C}\right)$.

Patch pipettes were drawn from $75 \mu \mathrm{l}$ of hematocrit tubing (VWR Scientific, San Francisco, CA) using a two-stage electrode puller. The pipette tips were $2 \mu \mathrm{m}$ in diameter and had open-tip resistances between 4 and $8 \mathrm{M} \Omega$ (DC). Resistance was compensated with the pipette submerged in the grounded bath using a standard "bridge balance" adjustment. Pipettes were filled with intracellular pipette solution that contained (in mM): $105 \mathrm{~K}$-gluconate, $35 \mathrm{KCl}, 5$ EGTA, $10 \mathrm{~K}$-HEPES, and 1 $\mathrm{MgCl}_{2}$. The $\mathrm{pH}$ of the solution was adjusted to 7.2 with $\mathrm{KOH}$, and osmolality was measured between 280 and 290 mOsm. The junction potential for this intracellular pipette solution was $7 \mathrm{mV}$ with reference to the grounded bath medium. All data are presented with correction for the junction potential.

Whole-cell voltage signals were recorded under current clamp using an Axoclamp 2B microelectrode amplifier (Axon Instruments, Foster City, CA). Tight seals $(>1 G \Omega)$ were established on the somata of visually identified NM neurons by applying slight negative pressure to the recording pipette on contact with the cell surface. The formation of gigaohm seals and the subsequent rupturing of the underlying membrane were monitored in voltage-clamp mode by measuring the resistive current resulting from a high-frequency, $-5 \mathrm{mV}$ pulse command. Stable recordings could be maintained for up to $1 \mathrm{hr}$. During recordings, we periodically monitored series resistance and capacitive currents to ensure good electrical access to the interior of the cell. Recordings were aborted if the membrane potential of a neuron depolarized to $-50 \mathrm{mV}$ or greater, and/or if a ruptured patch "resealed" and could not be ruptured again. Data were low-pass filtered at $10 \mathrm{kHz}$ and digitized with an ITC-16 (Instrutech, Great Neck, NY) at $20 \mathrm{kHz}$ for both on-line and off-line analysis. All recording protocols were written and run using the acquisition and analysis software Synapse, version 3.2 (Synergy, Bethesda, MD).

Concentric bipolar electrodes (Frederick Haer) with tip diameter of $0.5 \mathrm{~mm}$ were used for extracellular stimulation. The electrode was placed in the fiber tract dorsal to NM. Square electric pulses of $100 \mu \mathrm{sec}$ duration were delivered through a stimulus isolation unit and interval generator (WPI 1830). The stimulus was either a single pulse or a train of pulses at an intensity of $10-90 \mathrm{~V}$. Synaptic potentials were measured from a membrane potential of $-67 \pm 5 \mathrm{mV}$. Neurons with membrane potentials higher or lower were adjusted with DC current.

\section{RESULTS}

The goal of present study was to further understand the anatomical and functional properties of the GABAergic projections from the SON to NM and the other brainstem auditory nuclei. Fluorescent tracer was injected into the SON, NA, and NL for anterograde and retrograde labeling. The results showed that SON neurons project to the ipsilateral NM and to several other nuclei. To be inclusive, we present overall efferent and afferent connections of the SON with emphasis on its input to NM. We used a brain slice preparation to investigate the influence of the SON on NM. Stimulating SON fibers evoked long-lasting PSPs in NM neurons that were blockable by bicuculline, a potent antagonist of the $\mathrm{GABA}_{\mathrm{A}}$ receptor. We then describe a series of experiments aimed at evaluating the influence of the GABAergic input of SON on the membrane properties of NM neurons. 

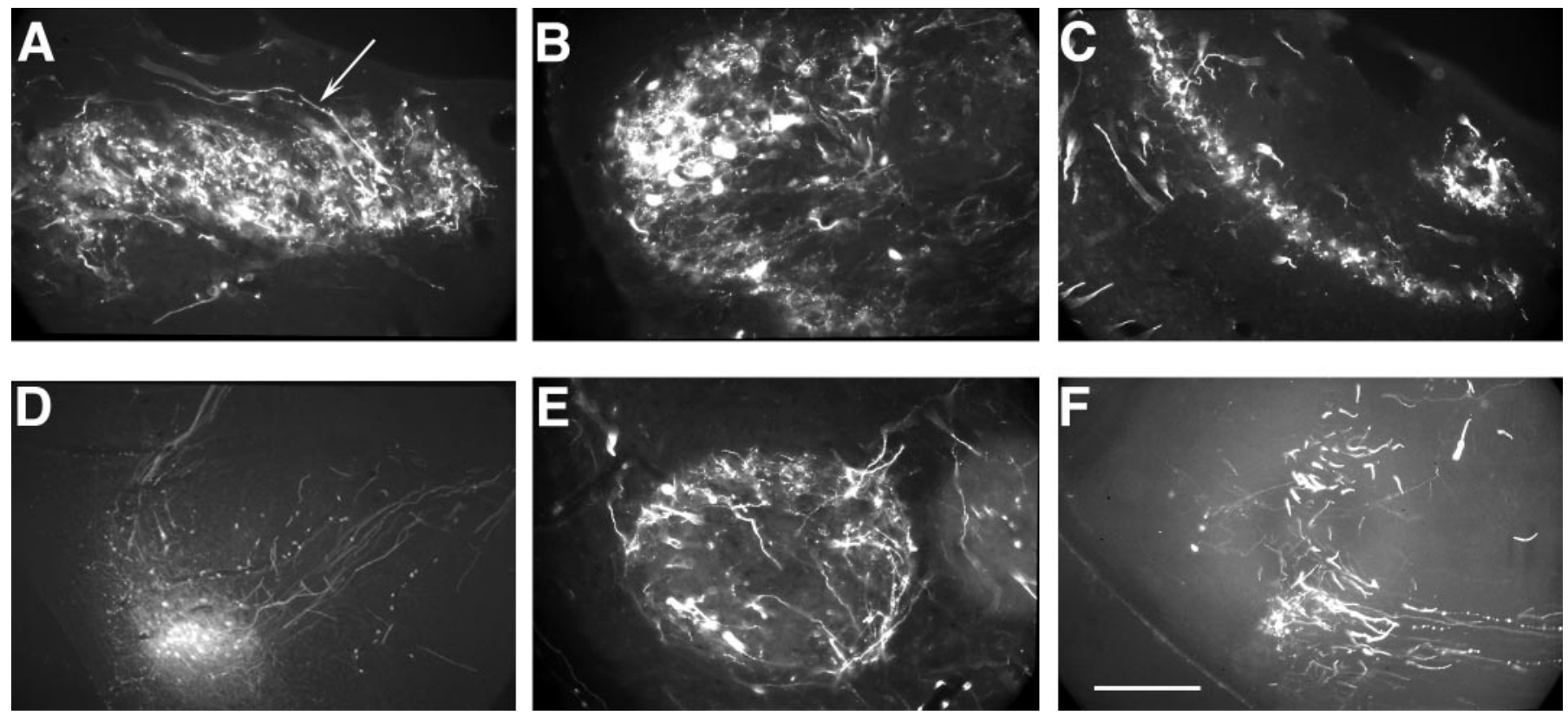

Figure 1. Micrographs of intensive labeling in the ipsilateral NM $(A)$, NA $(B)$, and NL $(C)$ after a focal injection in the ipsilateral SON $(D)$. Notice in NM the labeled SON fibers approach the nucleus from its dorsal area, as marked by an arrowhead. In NA and NL, especially in NA, there are many retrogradely labeled cell bodies. Furthermore, labeled SON cells at the injection site send projecting axons that form two fiber bundles as they emerge from the SON $(D)$. The dorsally oriented fiber bundle constitutes the ipsilateral projection and is responsible for all the anterograde labeling seen in this figure. The other fiber bundle form a contralateral projection to the contralateral SON $(E)$ and LLV $(F)$. Scale bar: $A-C, 45 \mu \mathrm{m} ; D, F, 115 \mu \mathrm{m}$.

Table 1. Percentage of total retrogradely labeled cells in the ipsilateral (i) and contralateral (c) NA, NM, and NL

\begin{tabular}{lllrrr} 
& iNA & iNM & iNL & cNA & cNM \\
\hline Injection was inside the SON (five cases) & $67 \%$ & 0 & $26 \%$ & $6 \%$ & 0 \\
Injection was in or spilled over to NTB and LLV (four cases) & $37 \%$ & 0 & $16 \%$ & $29 \%$ & 0
\end{tabular}

Tracer injection was either contained inside the superior olivary nucleus (SON, five cases) or spilled over to NTB and ventral LLV (four cases).

\section{SON connectivity}

Focal injection of rhodamine-conjugated to dextran (RCD) into the SON resulted in both retrograde and anterograde labeling in the ipsilateral NM, NL, and NA. The anterograde labeling represents the projecting terminals of the SON neurons, whereas retrograde labeling is the backfilling of cell bodies that innervate the SON. Figure 1 shows the prominent anterograde labeling in the ipsilateral NM, NL, and NA (Fig. $1 A-C$ ) and some retrograde labeling in the ipsilateral NA (Fig. $1 C$ ). For reasons described below, it was important to note that labeled SON fibers approached the ipsilateral NM from its dorsal margin. They branched extensively and formed numerous puncta that were distributed in patches or columns, a pattern similar to those of NM cell bodies (Fig. $1 A$ ).

In contrast to a previous study (Lachica et al., 1994), we never observed anterogradely labeled terminals or puncta in the contralateral NA, NM, and NL. Nevertheless, we did observe a contralateral projection from the SON to the contralateral SON and the ventral nucleus of the lateral lemniscus (LLV), a nucleus located immediately anterior to the SON (Fig. $1 E, F$ ). At the level of LLV and above, the contralateral SON projection converged with the terminal field of another fiber bundle; the ascending fibers from the contralateral NA and NL (Fig. $1 F$, pointed by the lower arrow). The labeling of this fiber bundle was attributable to retrograde filling of terminals from NA and NL innervating SON. These axons from NA and NL that terminate in the ipsilateral
SON also have collaterals that continue across the midline and innervate the nucleus of trapezoid body (NTB) and LLV before projecting further to other parts of the lateral lemniscus and midbrain. However, as shown in Figure $1 F$ and in the following experiments, these ascending fibers from NA and NL do not innervate the contralateral SON.

After RCD injection in the SON, there were always some retrogradely labeled cell bodies in ipsilateral NA and NL, but no labeled cells were observed in the ipsilateral NM. On average, more labeled cells were found in NA than in NL (Table 1). In all cases, there were some retrogradely labeled cell bodies in contralateral NA and NL, but they only constituted a small percentage of the total retrograde labeled cell bodies $(<7 \%)$. We found that the location of injection site influenced the number of retrograde labeled cells in the contralateral NA and NL. In contrast to the cases when the injection sites were inside the SON, injections that were made slightly anterior or ventral to the SON produced a substantial increase of labeling in the contralateral NA and NL (Table 1). The areas anterior and ventral to the SON are occupied by the LLV and NTB, respectively. Both nuclei receive input from the contralateral NA and NL (Westerberg and Schwarz, 1995). Given the close proximity of the SON to NTB and LLV, it was not surprising that a deposit outside the SON or spillover of tracer from the injection site would yield varying numbers of retrograde labeled cells in the contralateral NA and NL. 

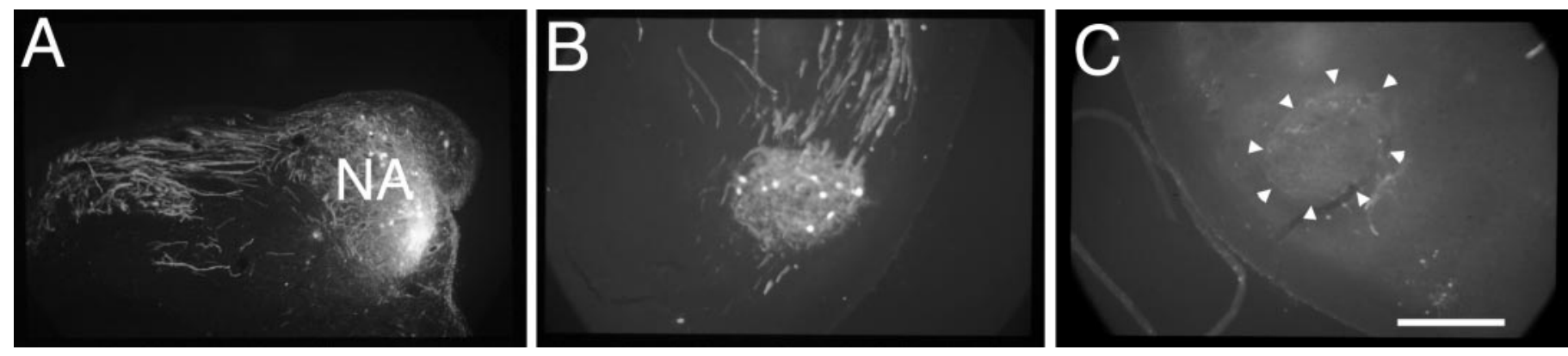

Figure 2. Anterograde and retrograde labeling in the ipsilateral $(B)$ and contralateral $(C)$ SON after a focal injection in the NA $(A)$. Notice that although there is intensive labeling in the ipsilateral SON, almost no labeling is present in the contralateral SON (arrowheads). Scale bar, $300 \mu \mathrm{m}$.

That the SON did not receive significant inputs from the contralateral NA and NL was further demonstrated by injecting tracer directly into the NA and NL. Eight animals received such injections (four in NL and four in NA). The anterogradely labeled NL and NA fibers were seen sending branches to the ipsilateral SON, but the same fibers never reached the contralateral SON. Figure 2 shows the retrograde and anterograde labeling in the ipsilateral $(B)$ and contralateral $(C) \mathrm{SON}$ after a focal $\mathrm{RCD}$ injection in the ipsilateral NA. Note that no anterograde labeling is seen in the contralateral SON. In addition, no labeled cell bodies are seen in the contralateral SON. In these eight animals, 441 cells were retrogradely labeled in the ipsilateral SON, but no cells were labeled in the contralateral SON.

In summary, based on the anterograde and retrograde labeling data we conclude that the SON projects to five nuclei in the auditory brainstem, the ipsilateral NM, NL, and NA and the contralateral SON and LLV. It receives inputs from three nuclei, the ipsilateral NL, NA, and contralateral SON.

\section{The reciprocal connections between the SON and ipsilateral NA and NL}

The data presented above depicted reciprocal connections between the SON and ipsilateral NA and NL. As to the ipsilateral NM, it only received input from the SON but did not project back to the SON. This organization was clearly shown in another set of experiments. In these experiments, we mixed RCD solution with $1 \%$ kainic acid. This concentration of kainic acid has been shown to kill cell bodies but spare passing fibers or innervating terminals (Glenn and Kelly, 1992). Thus, because SON cells are killed in this experiment, we expected only retrograde labeling in NA and NL. After injecting a mixture of kainic acid and RCD into SON, we compared the labeling patterns with those seen with injection of RCD alone (Fig. 1, no kainic acid). As shown in Figure 3D, after the mixture was injected into the SON, no labeled SON cells were seen at the injection site as with injections of RCD alone (Fig. 1D). In the ipsilateral NA and NL, there were many retrogradely labeled cell bodies (Fig. $3 A, C$ ) but no labeled terminal axons or puncta. In NM, there were neither retrogradely labeled cells nor the dense network of terminals and puncta, in contrast to injections of RCD alone (compare Figs. $3 B, 1 A$ ). The experiments also confirmed that the terminals and puncta seen in the injections of RCD alone were indeed from SON projection and not from axons passing through or near the SON.

\section{Evoked synaptic responses in NM neurons}

One distinguishing feature of NM neurons is outward rectification, as illustrated by Figure $4 A$. That is, the voltage response to hyperpolarizing current injection is much greater than that to
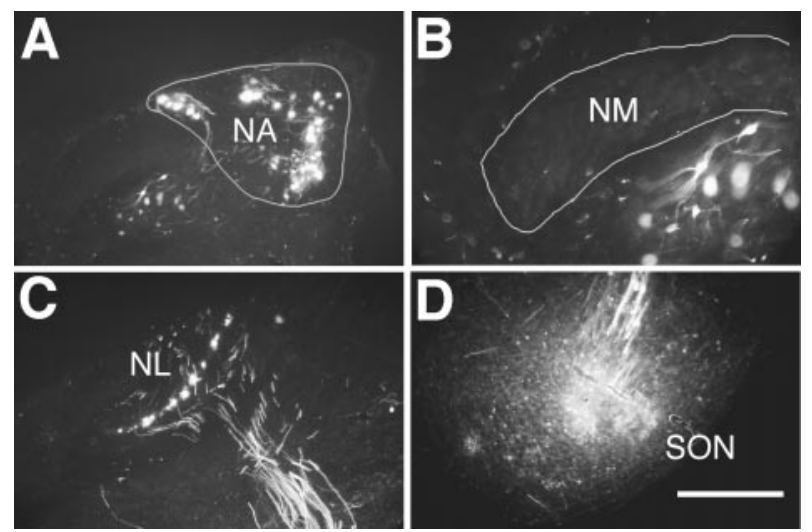

Figure 3. The labeling pattern seen in the ipsilateral NA $(A)$, NM $(B)$, and NL $(C)$ after a mixed solution of RCD and kainic acid was injected in the ipsilateral SON $(D)$. Comparing this result to the labeling pattern seen in normal injection (Fig. 1), kainic acid killed all SON neurons at the injection site. In NA and NL, there are no labeled terminals or punctate endings, cell bodies are retrogradely labeled. In NM, there are no labeled terminals or punctate endings, or retrogradely labeled cell bodies $(B)$. Labeled cells below NM in $B$ are retrogradely labeled NL neurons. Scale bar: $A, C, D, 300 \mu \mathrm{m} ; B, 120 \mu \mathrm{m}$.

depolarizing current injection of the same magnitude. Outward rectification is evident in the voltage range near resting potential, and is attributable to a $\mathrm{LVA} \mathrm{K}^{+}$conductance that is rapidly recruited. For this reason, the LVA conductance plays an important role in regulating the integration of synaptic inputs. (Reyes et al., 1994; Zhang and Trussell, 1994b; Koyano et al., 1996; Rathouz and Trussell, 1998). Suprathreshold currents never evoke more than one action potential, followed by a depolarized plateau, regardless of the current magnitude or amount of depolarization.

After briefly describing the various responses of NM we encountered when delivering electrical stimuli, we will focus on the responses of NM to stimulation of SON fibers and show how SON input can influence the firing of NM neurons. Synaptic responses of NM neurons were elicited by a stimulating electrode positioned just dorsal to NM. This site was chosen because SON fibers traverse this area before entering NM, and stimulation at this site was the most effective in evoking SON inhibition relative to other areas. However, in addition to SON fibers, this region also contains VIII nerve excitatory afferents and the axons of NM neurons (Young and Rubel, 1983). Therefore, several components could be evoked by stimulating this area, as shown in Figure $4 B-D$. In some cells, stimulation evoked excitatory orthodromic synaptic responses that were attributable to activation of V III 
Figure 4. Whole-cell recording of firing behavior and responses to electrical stimuli. In a representative NM neuron, responses to $100 \mathrm{msec}$ steps of injected current from -0.3 to $0.5 \mathrm{nA}$ illustrate the strong outward rectification typical of NM neurons $(A)$. Above threshold, NM neurons only fired one action potential at the onset of each current step, regardless of the size of the current step or the amount of depolarization. $B-D$ show responses in three different neurons evoked by stimulating fibers dorsal to NM with a $100 \mathrm{~Hz}$ train of shocks. In $B$, orthodromic action potentials were evoked by activation of VIII nerve fibers. These responses had latencies between 0.5 and $1 \mathrm{msec}$ and could fail when driven at rates of $\geq 100 \mathrm{~Hz}$, revealing the underlying EPSPs (asterisk). In $C$, antidromic action potentials were evoked when the axon of the NM neuron being recorded was activated, producing spikes with no measurable latency. $D$, An example showing a slow, summing depolarization of $5-10 \mathrm{mV}$ that was evoked along with orthodromic action potentials during a stimulus train. Note that the action potentials became progressively smaller in amplitude during the train as the baseline became more depolarized and failed altogether by the fourth stimulus. Stimulus artifacts in this and subsequent figures are truncated for clarity.
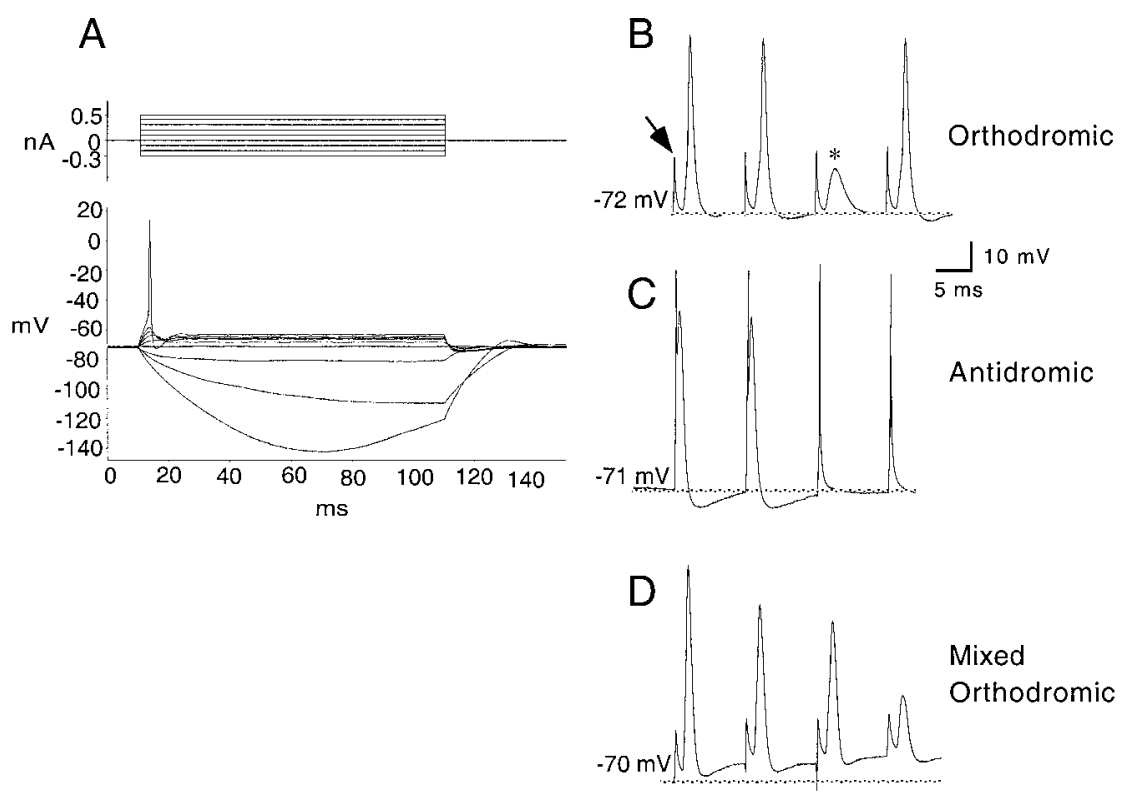

nerve afferent fibers (Fig. 4B). The response could be an action potential or EPSP (asterisk) and typically had a $0.5-1 \mathrm{msec}$ latency after stimulation (arrow indicates shock artifact). Other cells showed antidromic action potentials that were attributable to activation of NM neurons' own axons. These were distinguished from orthodromic responses in that EPSPs were never evoked by the stimulus, and spikes occurred with no measurable latency. Commonly, the leading edge of the antidromic action potentials merged with the shock artifact (Fig. 4C). In other cases, electric stimulation produced complex responses composed of two orthodromic components. As shown in Figure 4D, suprathreshold responses were accompanied by a slow depolarization that temporally summed with repetitive stimulation. During this train, orthodromically evoked spikes became progressively smaller in amplitude until ultimately failing, leaving only an EPSP response to the fourth stimulus.

By adjusting the stimulus amplitude or application of the glutamate receptor antagonists DNQX and AP-V, the slow orthodromic component could be isolated from excitatory responses. Figure 5, $A$ and $B$, illustrates the characteristics of slow PSPs in two NM neurons. In each neuron, responses to four successive shocks were recorded and superimposed. At a constant stimulus level, the amplitude and duration of PSPs fluctuated, possibly because of the variable number of fibers activated with each stimulus. For instance, the neuron shown in Figure $5 \mathrm{~A}$ responded to $30 \mathrm{~V}$ shocks with PSPs between 3 and $6 \mathrm{mV}$ in amplitude and $40-50 \mathrm{msec}$ in duration. One stimulus failed to elicit any response at all. Assuming that these responses were mediated by $\mathrm{GABA}_{\mathrm{A}}$ receptors, depolarizing responses were expected, because the reversal potential for $\mathrm{Cl}^{-}$under our recording conditions was $-34 \mathrm{mV}$. In the neuron shown in Figure $5 B$, a stimulus level of 50 $\mathrm{V}$ evoked PSPs with a range of amplitudes and a more rapid decay. The decay of PSPs was quantified in recordings from eight neurons by measuring the time required for PSPs to decay to 50\% of their peak amplitude. Figure $5 C$ shows that these decay values varied between 2 and $25 \mathrm{msec}$, but were mostly in the range between 5 and $20 \mathrm{msec}$. For comparison, the decay of EPSPs in five different neurons is plotted on the same graph; note the comparatively large amplitude and rapid decay of these responses. The variation in PSP decay we observed was at least partly attributable to variability in the input resistance among neurons, either because of size of the cells or the amount and activation of LVA currents present.

Close examination of the recordings revealed that larger PSPs did not decay smoothly; there was an initial rapid phase of repolarization followed by a slow phase. The initial rapid phase was absent in smaller PSPs, as shown in Figure $5 A$. The rapid phase of repolarization is shown in another neuron in Figure $5 D$, in which a downward inflection follows the peak of the PSP (arrow). Because this phenomenon occurred only with larger depolarizations, it was likely that LVA outward currents were involved in hastening the repolarization, because these currents activate near resting potential (Reyes et al., 1994; Koyano et al., 1996; Rathouz and Trussell, 1998). If this were the case, then hyperpolarizing the membrane potential would prevent the activation of LVA channels during the PSP, and thus its decay should occur smoothly. As shown by the darker trace in Figure 5E, a PSP evoked from a membrane potential held at $-93 \mathrm{mV}$ indeed showed a smoother decay without the rapid component. Previous studies have established that the onset of LVA activation in NM neurons can be observed in responses to subthreshold, depolarizing current injection. These responses are characterized by an initial depolarized voltage peak that is followed by a less depolarized plateau, reflecting the initial "passive" depolarization before the rapid activation of LVA outward currents (Zhang and Trussell, 1994b). In Figure 5F, we superimposed the waveform of a PSP on a voltage response of approximately the same amplitude evoked by direct current injection in the same neuron. Note the similarity in the timing of the onset of hyperpolarization in both waveforms. In summary, these experiments provide evidence that under our recording conditions, individual PSPs evoked by stimulating SON inputs activate some $\mathrm{LVA} \mathrm{K}^{+}$channels in addition to GABAergic channels.

These PSPs could undergo temporal summation evoked by trains of stimuli. A $100 \mathrm{~Hz}$ train of 10 stimuli at $60 \mathrm{~V}$ was delivered to another neuron. Figure $5 G$ shows that slow PSPs summed to produce a long-lasting depolarization with an amplitude of $\sim 15 \mathrm{mV}$ and a duration that outlasted the stimulus train by $>100 \mathrm{msec}$. Note change in time scale. For six neurons stimulated at $100 \mathrm{~Hz}$ for $100 \mathrm{msec}$, summation led to a maximum 
A
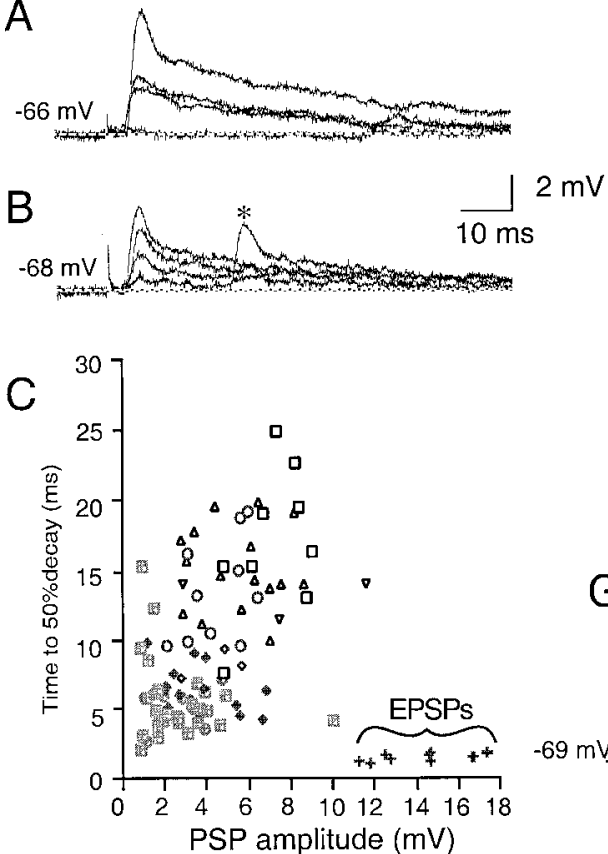

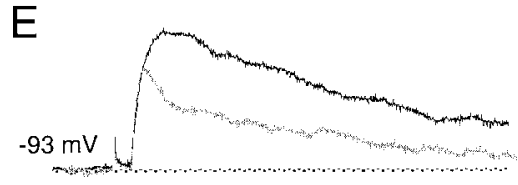

D
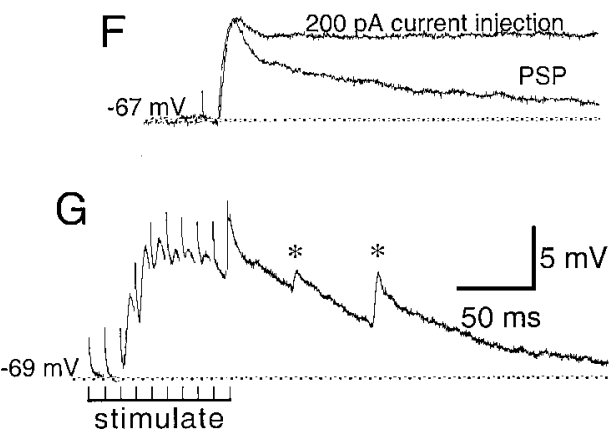

Figure 5. Slow, depolarizing PSPs evoked in NM neurons. In $A$ and $B$, responses to four successive shocks are superimposed for two neurons. Notice that amplitude of responses fluctuates between trials. In $C$, the amplitudes and decay characteristics are plotted for 76 evoked PSPs recorded in eight neurons. Each neuron is represented by a different symbol. For comparison, the amplitudes and decay of 10 evoked EPSPs in five neurons is shown (crosses). Note the consistently larger amplitude and faster decay of EPSPs. In $D$ and $E$, the recruitment of LVA outward currents by slow PSPs is shown in another neuron. A PSP evoked from resting potential $(-67 \mathrm{mV})$ showed a rapid phase to its repolarization ( $D$, arrow). PSPs evoked from a membrane potential of $-93 \mathrm{mV}$ did not display this rapid phase in their repolarization $(E)$. In $F$, a PSP from the same neuron as $D$ and $E$ superimposed on a response to a step of injected current $(+200 \mathrm{pA})$. The two records were selected to match in peak amplitude. Resting potential was $-67 \mathrm{mV}$ for both records. The onset of rapid hyperpolarization in the PSP was similar to the onset of LVA as revealed by the "sag" in the voltage response to current injection. In $G$, temporal summation of PSPs is shown for the neuron shown in $B$. Stimulating with a train of 10 shocks at $100 \mathrm{~Hz}$ resulted in temporal summation of slow PSPs that exceeded $10 \mathrm{mV}$ and decayed over $\sim 200$ msec. For $B$ and $G$, an asterisk indicates spontaneous PSPs. Stimulus intensity was $30 \mathrm{~V}$ for the neuron in $A, 50 \mathrm{~V}$ for the neuron in $B, 40 \mathrm{~V}$ for the neuron in $D-F$, and $60 \mathrm{~V}$ for the neuron in $G$.

depolarization of $11.9 \mathrm{mV}( \pm 3.4)($ mean $\pm \mathrm{SE})$, and time-to-peak depolarization occurred at $70 \mathrm{msec}( \pm 13.6)$ relative to the onset of the train. The decay of the depolarized plateau was more variable: the mean time to decay by $50 \%$ of the peak depolarization was $89.5 \mathrm{msec}( \pm 40.6)$. This variability in decay reflects our observation that in some neurons, stimulation with trains of shocks led to a temporary increase in the frequency of spontaneous PSPs (e.g., PSPs indicated by an asterisk in Fig. 5G), which has been observed by others ( $\mathrm{Lu}$ and Trussell, 2000) and is attributed to $\mathrm{Ca}^{2+}$ accumulation in GABAergic terminals.

\section{Slow synaptic responses are GABAergic}

Slow synaptic responses were blocked by bicuculline, a potent antagonist of $\mathrm{GABA}_{\mathrm{A}}$ receptors $(n=8$ cells). As shown in Figure 6 , in response to a $100 \mathrm{~Hz}$ train of pulses at $70 \mathrm{~V}$, the $\mathrm{NM}$ neuron responded with slow PSPs that summed and lasted beyond the duration of train pulses (Fig. 6A). Addition of DNQX (40 $\mu \mathrm{M})$ and APV $(100 \mu \mathrm{M})$, blockers of glutamate receptor-mediated EPSPs, to the bath media did not affect the amplitude or duration of the response (Fig. 6B). When bicuculline was added, the response was completely abolished (Fig. $6 C$ ), but recovered after 5-10 min of washout (Fig. 6D). Together, these data demonstrated that slow PSPs evoked in NM were attributable to direct activation of GABAergic fibers but not the glutamatergic inputs from VIII nerve fibers.

\section{GABAergic input affects firing of NM neurons}

Stimulating GABAergic inputs blocked action potentials in NM neurons $(n=6)$. This effect was demonstrated by activating the GABAergic fibers while evoking action potentials in single NM neurons by intracellular current injection. This paradigm is shown for one cell in Figure $7 A$. Under control conditions, a train of $3 \mathrm{msec}, 1.0 \mathrm{nA}$ current pulses was injected into a NM neuron at a $20 \mathrm{msec}$ interval. When the neuron was at rest, current injections reliably evoked action potentials, as illustrated by the first two spikes in Figure $7 A$. After the second spike, GABAergic fibers were activated by a train of 10 shocks at $100 \mathrm{~Hz}$, and PSPs (e.g., arrow) summed to produce a slow depolarization of the baseline membrane potential. During the depolarization, spikes were inhibited, revealing the underlying electrotonic responses to each current pulse injected (Fig. 7A, arrowhead). The onset of inhibition and its time course was similar to the envelope of the summed synaptic response.

SON input activated a GABAergic conductance, and the resulting depolarization also recruited $\mathrm{LVA} \mathrm{K}^{+}$channels. The activation of $\mathrm{LVA} \mathrm{K}^{+}$channels alone could have been inhibitory by shunting injected current. To test whether opening of $\mathrm{LVA} \mathrm{K}^{+}$ channels was sufficient to inhibit spikes, we directly depolarized the neuron with injected current to the same membrane potential reached during stimulation of GABAergic fibers $(n=5$; Fig. $7 B)$. Direct depolarization to the same level as that during GABAergic activation did not inhibit action potentials, indicating that opening of LVA channels alone was insufficient to account for the inhibitory effects seen.

The inhibition of action potentials could be overcome by increasing excitatory drive (Fig. $7 C, D$ ). In another neuron, spikes evoked with current pulses of $1.0 \mathrm{nA}$ and $2 \mathrm{msec}$ duration could be inhibited by stimulating SON inputs (Fig. $7 C$ ). By increasing the injected current amplitude to $1.5 \mathrm{nA}$, spikes could be recovered during stimulation of the GABAergic fibers (Fig. 7D).

The inhibition of the firing of NM neurons was most likely the consequence of shunting, attributable in part to the opening of GABAergic $\mathrm{Cl}^{-}$channels and in part to opening of LVA channels. Consequently, the same amount of excitation (current injection in this case) would not produce sufficient depolarization for the cell to reach threshold. To evaluate the relative contributions of GABAergic channels and LVA channels to the shunting inhibition, we measured input resistance, under three conditions: at rest, during SON stimulation, and during membrane depolarization $(n=5)$. Voltage responses to low-amplitude $(-50$ to -150 pA), 50 msec hyperpolarizing pulses of current were recorded, 

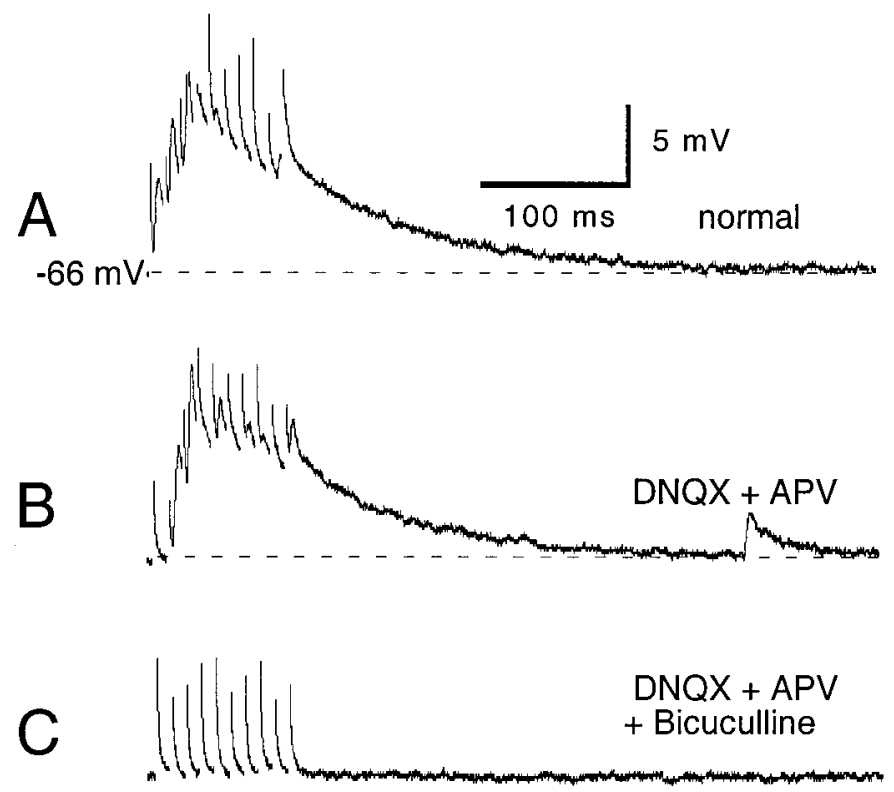

D

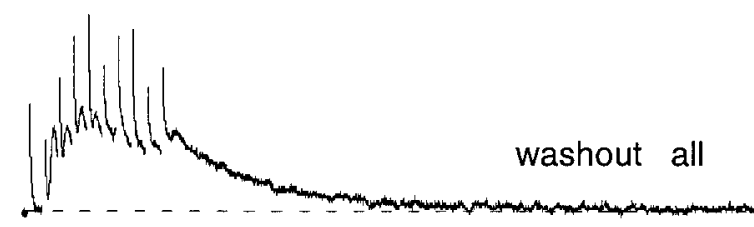

Figure 6. Slow PSPs are mediated by $\mathrm{GABA}_{\mathrm{A}}$ receptors. Summed responses to stimulation with 10 shocks at $100 \mathrm{~Hz}$ and $70 \mathrm{~V}(A)$. Response amplitude or duration were not altered in the presence of DNQX $(50 \mu \mathrm{M})$ and AP-V $(100 \mu \mathrm{M})(B)$. Adding $50 \mu \mathrm{M}$ bicuculline completely abolished the response $(C)$, which could be recovered within 5-10 $\mathrm{min}(D)$.

and 10 responses were averaged to give a mean voltage response to a given current amplitude. Input resistance $\left(R_{\text {in }}\right)$ was calculated as $V-I$. An example case is illustrated in the left panel of Figure 8 , where the NM neuron had an input resistance of $67 \mathrm{M} \Omega$ at resting potential, which in all cases was adjusted to $-67( \pm 5 \mathrm{mV})$. Under this condition, we assumed that GABAergic and LVA conductances were minimal. Although GABAergic inputs were stimulated (Fig. 8, middle panel), $R_{\text {in }}$ was reduced to $31 \mathrm{M} \Omega$, and this change was probably attributable to opening of GABAergic channels and LVA $\mathrm{K}^{+}$channels. To estimate the contribution of the LVA conductance to shunting, $R_{\text {in }}$ was measured in the same neurons while they were directly depolarized from resting potential with a baseline of positive current (Fig. 8, right panel). As expected, direct depolarization of the NM neuron also reduced the input resistance, but not to the same extent that it was reduced during GABAergic input. For this cell, depolarization alone reduced $R_{\text {in }}$ to $48 \mathrm{M} \Omega$. For five NM neurons, stimulating GABAergic fibers reduced $R_{\text {in }}$ by an average of $61 \%$ from a mean $( \pm \mathrm{SD})$ of $75( \pm 38) \mathrm{M} \Omega$ to a mean $( \pm \mathrm{SD})$ of $28( \pm 20) \mathrm{M} \Omega$. In the same neurons, depolarization alone reduced $R_{\text {in }}$ by an average of $41 \%$, indicating that approximately two-thirds $(65 \%)$ of the change in $R_{\text {in }}$ during stimulation of SON input is attributable to LVA channels. In summary, GABAergic input evoked a shunting inhibition in NM neurons that was partly attributable to GABAactivated channels and partly to low-threshold voltage activated $\mathrm{K}^{+}$channels.

\section{DISCUSSION}

The present study addresses the functional significance of the SON in the avian auditory brainstem, and more generally, the role of inhibition in auditory processing. We show that the SON projects to the ipsilateral NM, NA, and NL as well as SON and LLV on the contralateral side. In return, the SON receives inputs from the ipsilateral NA and NL and contralateral SON. We further demonstrate that the GABAergic projection from the SON to NM can be blocked by bicuculline, a potent antagonist for $\mathrm{GABA}_{\mathrm{A}}$ receptors and that SON-mediated GABAergic inhibition decreases membrane input resistance through a shunting effect. The mechanism is unique in that it involves the cooperative action between $\mathrm{GABAergic} \mathrm{Cl}^{-}$and $\mathrm{LVA} \mathrm{K}^{+}$channels. This reduced input resistance makes NM neurons less excitable, and, as discussed below, may lead to the enhancement of phase locking by NM neurons. One possible problem in our comparisons of anatomy in hatchlings to physiology in embryos is that at least one aspect of the GABAergic innervation of NM, terminal density, is still maturing in the first 2 weeks after hatching (Code et al., 1989). However, intrinsic properties of NM neurons are similar in hatchlings and late embryos (Reyes et al., 1994; Zhang and Trussell, 1994b).

\section{SON feedback is primarily ipsilateral}

The anatomical aspects of our study were intended to both confirm previous findings and clarify one issue that is inconsistent in previous studies: whether or not there are reciprocal connections between the SON and the contralateral NA and NL. Several studies describe a contralateral input to the SON from NA and NL, but the reported strength of this projection varies greatly (Lachica et al., 1994; Westerberg and Schwarz, 1995). Whereas the discrepancy may be in part because of the different tracers used, our data and those of Westerberg and Schwarz (1995) suggest that most of the variability may be accounted for by the size of the tracer deposit. The SON is closely apposed to two other nuclei, LLV and NTB, which both receive input from the contralateral NA and NL (Takahashi and Konishi, 1988; Westerberg \& Schwarz, 1995). Consequently, any spillover to LLV and NTB from a large injection would produce retrograde labeling in the contralateral NA and NL. Lachica et al. (1994) did not distinguish among the SON and these neighboring nuclei, and the injection sites in that study were usually large enough to extend beyond the borders of SON.

\section{SON may act to equalize the activity in the ipsilateral and contralateral NM}

The unilateral connectivity between SON and NM, NA, and NL that we observed has significant implications for the role that this circuitry plays in processing binaural information. Previous studies have demonstrated that SON neurons fire at rates that are graded with the amount of excitatory drive (Moiseff and Konishi, 1983; Yang et al., 1999) and are anatomically and physiologically suited for spatial and temporal summation. These findings are consistent with a gain control mechanism proposed previously (Lachica et al., 1994; Peña et al., 1996). The hypothesis is that without inhibition, high sound levels would strongly drive each $\mathrm{NM}$, and the high firing rate from either side might lead to "false alarms" in NL neurons. That is, coincident firing of many NM fibers from either side alone can sum and evoke action potentials in NL neurons, deteriorating the ability of NL to discriminate binaural coincidences from strong unilateral excitation. SON potentially can prevent strong monaural excitation because inhibi- 


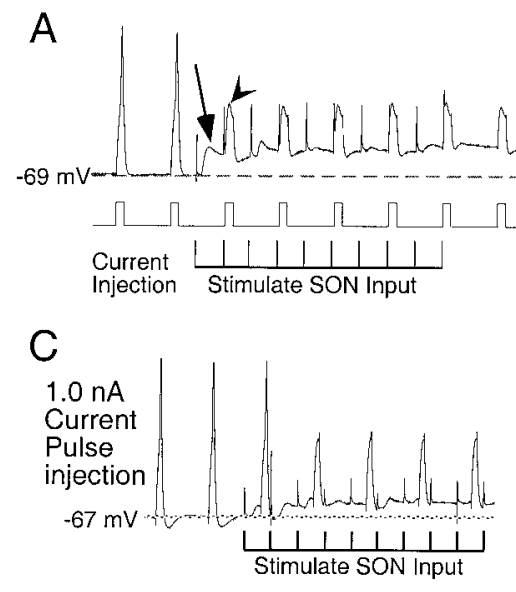

Figure 7. The depolarizing, GABAergic input of SON blocked action potentials in NM. In $A$, a series of action potentials were evoked by injecting $3 \mathrm{msec}$ current pulses of $1.0 \mathrm{nA}$ at $20 \mathrm{msec}$ intervals. After the second current pulse, SON fibers were stimulated with 10 shocks at $100 \mathrm{~Hz}$ and $70 \mathrm{~V}$. Notice, in response to stimulating SON input, NM neurons produced PSPs (arrow) that summed and depolarized the membrane potential by $15 \mathrm{mV}$. Correspondingly, the same current injection failed to elicit action potentials during and after the stimulus train. The time course of recovery correlated with the duration of depolarization evoked by stimulating SON inputs. In the same neuron, depolarizing the membrane potential without stimulating SON inputs did not block the generation of action potentials $(B)$. The neuron was depolarized with a steady current to the same level as that evoked by stimulating SON inputs. $C$ and $D$ show that inhibition can be overcome by increasing excitatory drive. In another neuron, action potentials inhibited by stimulating SON input $(C)$ could be rescued by increasing the amount of depolarizing current injected $(D)$. Protocol used was similar to that in $A$ and $B$, except current pulses were $2 \mathrm{msec}$ in duration. tion from SON is proportional to the ipsilateral sound level and thus would reduce the activity of NM correspondingly. The unilateral projection from SON to NM allows separate control of $\mathrm{NM}$ activity on each side of the brain. Thus, the side receiving the strongest excitatory drive also receives proportionally greater inhibition; any large disparity in NM firing between the two sides will be reduced. Furthermore, the inhibition to each side is not entirely independent because of the reciprocal innervation between the two SONs, making SON activity dependent on the ipsilateral sound level and the degree of inhibition provided by the opposite SON. In this way, similar firing rates in ipsilateral and contralateral NM could be maintained at a level appropriate for coincidence detection, even though sounds located off the midline generate interaural intensity disparities as well as timing disparities (Hyson et al., 1994).

\section{Functional implications for inhibition of NM neurons}

Inhibition from SON may also influence phase locking in NM neurons via two different mechanisms. The first mechanism is through the activation of $\mathrm{GABA}_{\mathrm{B}}$ receptors. Brenowitz et al. (1998) showed that $\mathrm{GABA}_{\mathrm{B}}$-mediated attenuation of transmitter release prevents depression, enabling relatively uniform EPSC amplitudes at high stimulus frequencies. During trains of stimuli, the regularity of EPSC amplitude conserves phase information by making the relative timing between presynaptic and postsynaptic spikes more uniform; i.e., spike threshold is crossed at the same time with each EPSC.

The second mechanism is through postsynaptic inhibition of the NM cell directly via activation of $\mathrm{GABA}_{\mathrm{A}}$ receptors, which should actually enhance the phase locking precision of NM neurons relative to their auditory nerve inputs. The postsynaptic
GABAergic inhibition causes a substantial decrease in input resistance, thereby shunting inward currents and preventing NM cells from reaching threshold with currents that are suprathreshold in the absence of inhibition. The hypothesis we now advance is that with SON inhibition, currents generated by one afferent terminal are subthreshold, and only by coincident input from two or more afferents are excitatory currents suprathreshold. It is noteworthy that in our study, stimulating SON input blocked action potentials in NM that were evoked with current pulses of 1.0-1.5 nA. These current amplitudes are comparable to the amplitudes of EPSCs evoked by stimulation of single afferent fibers of NM (Brenowitz et al., 1998). The requirement of coincident subthreshold inputs improves phase locking by producing a tighter registration of discharges at a particular phase of the signal than would occur if each input was suprathreshold, an idea that has been proposed previously by Carney (1992), Rothman et al. (1993), Joris et al. (1994), and Rothman and Young (1996) to account for the improved phase locking of bushy cells in the cochlear nucleus of mammals over that of auditory nerve fibers. Comparisons of phase locking by NM neurons and auditory nerve fibers in the barn owl have reached conflicting conclusions on whether there is a similar enhancement of synchrony in avians (Sullivan and Konishi, 1984; Köppl, 1997).

\section{Why is SON-evoked GABAergic inhibition depolarizing?}

Depolarizing responses to GABA, although common in developing nervous systems, are rarely observed in the CNS of mature animals. In the present study, we obtained depolarizing GABAergic responses because our recording conditions gave rise to a $\mathrm{E}_{\mathrm{Cl} \text { - }}$ of $-34 \mathrm{mV}$; opening of $\mathrm{Cl}^{-}$channels allows $\mathrm{Cl}^{-}$efflux,

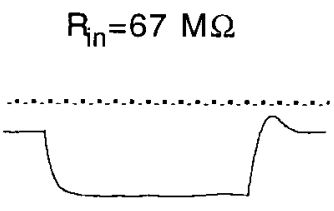

Resting

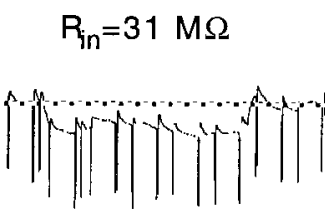

With SON Input

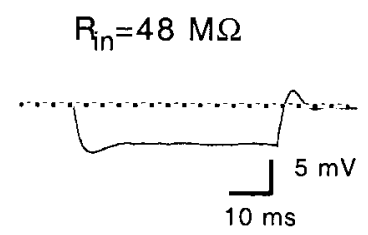

With Depolarization
Figure 8. Stimulating SON fibers lowered input resistance $\left(R_{\text {in }}\right)$ in NM neurons. $R_{\text {in }}$ was measured from averaged, peak voltage deflection during injection of a $-0.15 \mathrm{nA}, 50 \mathrm{msec}$ current pulse at rest (left panel), during stimulation of SON inputs (middle panel), and during direct depolarization equivalent to that reached during SON stimulation (right panel). Resting potential was $-66 \mathrm{mV}$, and SON input caused depolarization to $-62 \mathrm{mV}$. SON inputs were stimulated with a train of 30 shocks at $100 \mathrm{~Hz}$ and $60 \mathrm{~V}$. Ten sweeps were taken for averaging under each condition. Notice that depolarization alone reduces $R_{\text {in }}$ but not to the extent observed during stimulation of SON inputs. Dashed line indicates the level of depolarization reached during SON stimulation. 
depolarizing the cells. Nevertheless, depolarization by SON input probably reflects the normal polarity of GABAergic responses for neurons in NM, as depolarizing responses have been observed in 2-week-old hatchlings using methods that minimally disturbed intracellular $\left[\mathrm{Cl}^{-}\right.$] (Hyson et al., 1995).

The important consideration here is that the functional advantage that may result from depolarizing inhibition would not occur with the hyperpolarizing inhibition found more commonly in other systems. As has been suggested previously (Hyson et al., 1995), one advantage of having a depolarizing inhibition is to allow the recruitment of additional conductances that are activated slightly above resting potential. GABA-activated $\mathrm{Cl}^{-}$channels appear to act synergistically with LVA channels in NM neurons; $\mathrm{Cl}^{-}$efflux through GABAergic channels depolarizes the neuron and thereby recruits LVA channels. Opening of LVA channels contributed approximately two-thirds of the change in input resistance we measured when SON fibers were stimulated. Thus, depolarizing inhibition is particularly effective because of the recruitment of $\mathrm{LVA} \mathrm{K}^{+}$channels. Indeed, one may argue that this strategy is more effective for shunting inward currents than a GABAergic response that is hyperpolarizing or has no effect on membrane potential. This appears to give the SON greater leverage in adjusting excitability and perhaps the temporal characteristics of NM neurons as well.

\section{REFERENCES}

Boord RL (1969) The anatomy of the avian auditory system. Ann NY Acad Sci 167:147-155.

Brenowitz S, David J, Trussell LO (1998) Enhancement of synaptic efficacy by presynaptic $\mathrm{GABA}_{\mathrm{B}}$ receptors. Neuron 20:135-41.

Carney LH (1992) Modeling the sensitivity of cells in the anteroventral cochlear nucleus to spatiotemporal discharge patterns. Philos Trans R Soc Lond B Biol Sci 336:403-406.

Carr CE, Boudreau RE (1991) Central projections of auditory nerve fibers in the barn owl. J Comp Neurol 314:306-318.

Carr CE, Boudreau RE (1993) Organization of the nucleus magnocellularis and the nucleus laminaris in the barn owl: encoding and measuring interaural time differences. J Comp Neurol 334:337-355.

Carr CE, Konishi M (1988) Axonal delay lines for time measurement in the owl's brainstem. Proc Natl Acad Sci USA 85:8311-8315.

Carr CE, Konishi M (1990) A circuit for detection of interaural time differences in the brainstem of the barn owl. J Neurosci 10:3227-3246.

Carr CE, Fujita I, Konishi M (1989) Distribution of GABAergic neurons and terminals in the auditory system of the barn owl. J Comp Neurol 286:190-207.

Code RA, Burd GD, Rubel EW (1989) Development of GABA immunoreactivity in brainstem auditory nuclei of the chick: ontogeny of gradients in terminal staining. J Comp Neurol 284:504-518.

Code RA, Durham D, Rubel EW (1991) Effects of cochlear removal on GABAergic terminals in nucleus magnocellularis of the chicken. J Comp Neurol 301:643-654.

Fujita I, Konishi M (1991) The role of GABAergic inhibition in processing of interaural time difference in the owl's auditory system. J Neurosci 11:722-739.

Glenn SL, Kelly JB (1992) Kainic acid lesions of the dorsal nucleus of the lateral lemniscus: effects on binaural evoked responses of rat auditory cortex. J Neurosci 12:3688-3699.

Hackett JT, Jackson H, Rubel EW (1982) Synaptic excitation of the second and third order auditory neurons in the avian brain stem. Neuroscience 7:1455-1469.

Hyson RL, Rubel EW (1989) Transneuronal regulation of protein synthesis in the brain-stem auditory system of the chick requires synaptic activation. J Neurosci 9:2835-2845.

Hyson RL, Overholt EM, Lippe WR (1994) Cochlear microphonic measurements of interaural time differences in the chick. Hear Res 81:109-118.

Hyson RL, Reyes AD, Rubel EW (1995) A depolarizing inhibitory response to GABA in brainstem auditory neurons of the chick. Brain Res 667:117-126.

Jhaveri S, Morest DK (1982a) Neuronal architecture in nucleus magno- cellularis of the chicken auditory system with observations on nucleus laminaris: a light and electron microscope study. Neuroscience 7:809-836

Jhaveri S, Morest DK (1982b) Sequential alterations of neuronal architecture in nucleus magnocellularis of the developing chicken: a Golgi study. Neuroscience 7:837-853.

Joris PX, Smith PH, Yin TC (1994) Enhancement of neural synchronization in the anteroventral cochlear nucleus II: responses in the tuning curve tail. J Neurophysiol 71:1037-51.

Joseph AW, Hyson RL (1993) Coincidence detection by binaural neurons in the chick brain stem. J Neurophysiol 69:1197-1211.

Köppl C (1997) Phase locking to high frequencies in the auditory nerve and cochlear nucleus magnocellularis of the barn owl, Tyto alba. J Neurosci 17:3312-3321.

Koyano K, Funabiki K, Ohmori H (1996) Voltage-gated ionic currents and their roles in timing coding in auditory neurons of the nucleus magnocellularis of the chick. Neurosci Res 26:29-45.

Lachica EA, Rübsamen R, Rubel EW (1994) GABAergic terminals in nucleus magnocellularis and nucleus laminaris originate from the superior olivary nucleus. J Comp Neurol 348:403-418.

Lu T, Trussell L (2000) Dynamics of GABAergic transmission in the avian cochlear nucleus. Association for Research in Otolaryngology Midwinter Meeting, St. Petersburg, FL, February.

Lu T, Oertel D, Trussell L (1997) GABA-evoked conductance in the avian cochlear nucleus. Soc Neurosci Abstr 23:1548.

Moiseff A, Konishi M (1983) Binaural characteristics of units in the owl's brainstem auditory pathway: precursors of restricted spatial receptive fields. J Neurosci 3:2553-2562.

Müller CM (1987) $\gamma$-aminobutyric acid immunoreactivity in brainstem auditory nuclei of the chicken. Neurosci Lett 77:272-276.

Overholt EM, Rubel EW, Hyson RL (1992) A circuit for coding interaural time differences in the chick brain stem. J Neurosci 12:1698-1708.

Parks TN (1981) Morphology of axosomatic endings in an avian cochlear nucleus: nucleus magnocellularis of the chicken. J Comp Neurol 203:425-440.

Parks TN, Rubel EW (1978) Organization and development of the brain stem auditory nuclei of the chicken. J Comp Neurol 180:439-448.

Peña JL, Viete S, Albeck Y, Konishi M (1996) Tolerance to intense sound of binaural coincidence detection in the owl's nucleus laminaris. J Neurosci 16:7046-7054.

Raman IM, Trussell LO (1992) The kinetics of the response to glutamate and kainate in neurons of the avian cochlear nucleus. Neuron 9:173-186.

Raman IM, Zhang S, Trussell LO (1994) Pathway-specific variants of AMPA receptors and their contribution to neuronal signaling. J Neurosci 14:4998-5010.

Ramón y Cajal S (1971) The acoustic nerve: its cochlear branch or cochlear nerve. Translated from: Histologie du systèmè nerveux de i'homme et des vertèbrès, Tome I, pp 774-838:1952. National Technical Information Service Publication No. PB-205 473.

Rathouz M, Trussell LO (1998) Characterization of outward currents in neurons of the avian nucleus magnocellularis. J Neurophysiol $80: 2824-2835$

Reyes AD, Rubel EW, Spain WJ (1994) Membrane properties underlying the firing of neurons in the avian cochlear nucleus. J Neurosci 14:5352-5364.

Reyes AD, Rubel EW, Spain WJ (1996) In vitro analysis of optimal stimuli for phase-locking and time-delayed modulation of firing in avian nucleus laminaris neurons. J Neurosci 16:993-1007.

Rothman JS, Young ED (1996) Enhancement of neural synchronization in computational models of ventral cochlear nucleus bushy cells. Audit Neurosci 2:47-62.

Rothman JS, Young ED, Manis PB (1993) Convergence of auditory nerve fibers onto bushy cells in the ventral cochlear nucleus: implications of a computational model. J Neurophysiol 70:2562-2583.

Rubel EW, Parks TN (1975) Organization and development of brain stem auditory nuclei of the chicken: tonotopic organization of n. magnocellularis and n. laminaris. J Comp Neurol 164:411434.

Sullivan WE, Konishi M (1984) Segregation of stimulus phase and intensity coding in the cochlear nucleus of the barn owl. J Neurosci 4:1787-1799.

Takahashi T, Konishi M (1988) The projections of nucleus angularis and nucleus laminaris to the lateral lemniscal nuclear complex of the barn owl. J Comp Neurol 274:212-238.

Trussell LO, Zhang S, Raman IM (1993) Desensitization of AMPA 
receptors upon multi-quantal neurotransmitter release. Neuron 10:1185-1196.

von Bartheld CS, Code RA, Rubel EW (1989) GABAergic neurons in brainstem auditory nuclei of the chick: distribution, morphology and connectivity. J Comp Neurol 287:470-483.

Warchol ME, Dallos P (1990) Neural coding in the chick cochlear nucleus. J Comp Physiol 166:721-734.

Westerberg BD, Schwarz DWF (1995) Connections of the superior olive in the chicken. J Otolaryngol 24:20-30.

Yang L, Monsivais P, Rubel EW (1999) The superior olivary nucleus and its influence on nucleus laminaris: a source of inhibitory feedback for coincidence detection in the avian auditory brainstem. J Neurosci 19:2313-2325.

Young SR, Rubel EW (1983) Frequency specific projections of individual neurons in chick brain stem auditory nuclei. J Neurosci 3:1373-1378.

Zhang S, Trussell LO (1994a) Voltage clamp analysis of excitatory synaptic transmission in the avian nucleus magnocellularis. J Physiol (Lond) 480:123-136.

Zhang S, Trussell LO (1994b) A characterization of excitatory postsynaptic potentials in the avian nucleus magnocellularis. J Neurophysiol 72:705-718. 